\title{
Vasa previa: a case report
}

\section{Ipsita Mohapatra*, Subha R. Samantaray, V. Naga Sindhuja}

Department of Obstetrics and Gynaecology, Prathima Institute of Medical Sciences, Telangana, India

Received: 01 August 2020

Accepted: 03 September 2020

\section{*Correspondence:}

Dr. Ipsita Mohapatra,

E-mail: demurerosy@gmail.com

Copyright: () the author(s), publisher and licensee Medip Academy. This is an open-access article distributed under the terms of the Creative Commons Attribution Non-Commercial License, which permits unrestricted non-commercial use, distribution, and reproduction in any medium, provided the original work is properly cited.

\begin{abstract}
Vasa previa is defined as a condition where fetal vessels traverse the membranes in the lower segment below the presenting part unsupported by placental tissue or umbilical cord. Rupture of the membranes leads to fetal exsanguinations and even neonatal death. The etiology is uncertain, but risk factors include bilobed or succenturiate lobed placenta, velamentous insertion of cord, placenta previa, pregnancies resulting from In vitro fertilization (IVF) and multiple pregnancies. We report here a case of 24 year old woman, G3A2 at 34 weeks of gestation and history of 2 previous spontaneous abortions with vasa previa which was successfully managed. Prenatal sonographic diagnosis has the potential to improve or prevent the poor obstetric and neonatal outcome associated with it.
\end{abstract}

Keywords: Vasa previa, Prenatal diagnosis, Fetal demise

\section{INTRODUCTION}

Vasa previa is defined as a condition where fetal vessels traverse the membranes in the lower segment below the presenting part unsupported by placental tissue or umbilical cord. Rupture of the membranes leads to fetal exsanguinations and even neonatal death. The fetal mortality from undiagnosed vasa previa ranges from $60-70 \% .^{1}$ It is the unexpected manner in which sudden fetal demise occurs that makes this complication one of the most dreaded and challenging events in modern obstetrics. However, the advent of ultrasonography in obstetric practice has made the diagnosis possible prenatally as early as second trimester. We present here a case of vasa previa which was successfully managed at our institute.

\section{CASE REPORT}

We report here a case of 24 year old woman, G3A2 at 34 weeks of gestation and history of 2 previous spontaneous abortions. She was referred to our hospital in view of bleeding per vaginum at 34 weeks. Routine anomaly scan done at 20 weeks of gestation reported normal fetal anatomy with low lying placenta. On admission her vitals were stable. She had no pallor, BP was $130 / 86 \mathrm{~mm}$ of $\mathrm{Hg}$ and pulse rate was $92 / \mathrm{min}$. Fetal heart rate was 142 beats/min. Admission NST was reactive. Per vaginal examination was not done. Repeat scan was done which revealed posterior low lying placenta with accessory lobe and features of vasa previa. Colour doppler was done for confirmation which showed multiple blood vessels over lower margin of placenta and crossing to succenturiate lobe suggestive of vasa previa (Figure 1).

Case was posted for emergency caesarean section in view of vasa previa with antepartum hemorrhage and delivered a single live female baby of weight $2 \mathrm{~kg}$. Intra operatively there was atonic post-partum haemorrhage which was controlled with uterotonics and B-lynch suturing. The placenta was examined. It was $700 \mathrm{~g}$ in weight. Unsupported blood vessels were found to traverse from main placental lobe to the succenturiate lobe (Figure 2). The blood loss was around $800 \mathrm{ml}$. The placenta with membranes and the umbilical cord were sent for histological examination. Placental histopathological examination revealed the presence of membranous cord anchorage. Placental chorionic villi had a normal 
histological structure with minimal intervillous fibrinoid structures and calcinates. No inflammation was detected in the chorionic plate, umbilical cord and membranes.

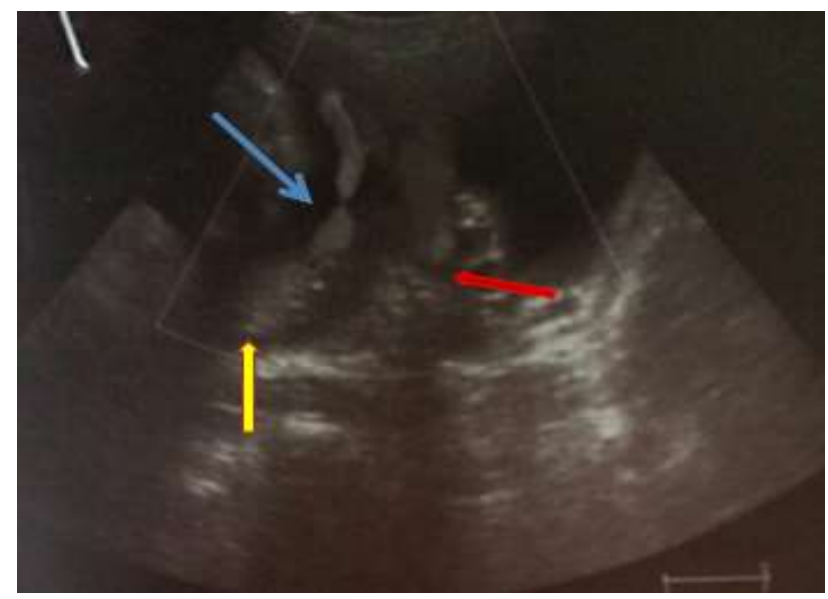

Figure 1: In the Doppler study blue arrow- fetal vessels crossing over OS, yellow arrow - posteriorly situated placenta, red arrow - internal OS

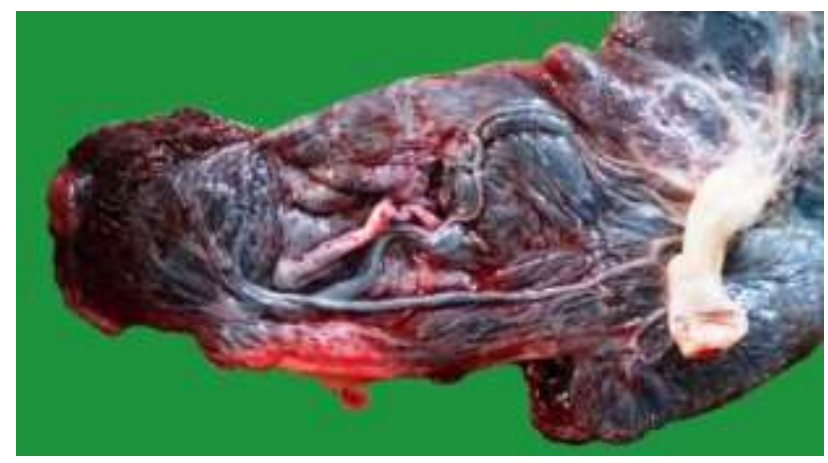

Figure 2: Gross appearance of placenta in which blood vessels traversing from main placental lobe to the succenturiate lobe

Post operatively she developed hypertension (blood pressure was measured to be $>160 / 110 \mathrm{~mm}$ of $\mathrm{Hg}$ on two occasions) for which we administered anti-hypertensives tablet nifedepin sustained release $20 \mathrm{mg}$ twice a day. 1 unit packed cell volume (PCV) and 1 unit Fresh frozen plasma (FFP) were given for correction of blood loss. Blood pressure gradually normalized and she was off anti-hypertensives on 5 th post-operative day. She was discharged under stable condition on 7 th post op day with a healthy baby.

\section{DISCUSSION}

Vasa previa is a rare condition occurring in 1 in 1275-5000 pregnancies. $^{2}$ The etiology is uncertain, but risk factors include bilobed or succenturiate lobed placenta, velamentous insertion of cord, placenta previa, pregnancies resulting from IVF and multiple pregnancies. ${ }^{3,4}$ There are two types of vasa praevia. Type 1 , when fetal blood vessels cross over the uterine OS, this corresponds to a velamentous insertion of the umbilical cord. Type 2, when fetal blood vessels are in the same location as type 1 but they run between the lobes of the placenta (bilobed or succenturiate lobed) as seen in present case. $^{5}$

Vasa previa is one of the rare causes of antepartum haemorrhage and hence patient should be educated about the importance of regular antenatal care and early detection of placenta praevia and rarely vasa previa. $^{6}$ Ultrasonographic prenatal detection of vasa previa has made a significant impact in the reduction of perinatal mortality and morbidity from this condition. Sonographic clues include identifying vessels overlying the internal OS, although it may be due to cord presentation. Vasa previa should be differentiated from cord presentation by observing their relative positions on repeated scans. The vessels change in position in cord presentation, where as in vasa previa the vessels remain on the same site on repeated scans. Vasa previa is confirmed if an aberrant vessel is seen to cross the internal OS. It has been suggested that because of association of vasa previa with placental abnormalities or velamentous cord insertion, bilobed and marginal placentas, an attempt must be made to look for umbilical cord insertion. Anomaly scan performed at 20 weeks may identify low placenta.This is an important risk factor for vasa previa and an attempt should be made to rule out vasa previa.

Vasa previa may clinically present as vaginal bleeding at rupture of membranes spontaneously or artificially and may be misinterpreted as heavy show. Whenever associated fetal heart abnormalities are there, one should have a suspicion of vasa previa.6 Vessel compression may result because of labour, digital pressure or pressure of presenting part which can lead to severe Cardiotocography (CTG) abnormalities like fetal heart decelerations, bradycardia, and sinusoidal heart rate pattern usually signifying fetal hemorrhage which may result in fetal demise. ${ }^{8,9}$ As the fetal blood volume is only $250 \mathrm{ml}$ at term, a loss of $60 \mathrm{ml}$ or more is sufficient to cause fetal exsanguination and demise. ${ }^{10}$ Prenatal diagnosis allow us to monitor bleeding symptoms closely and facilitates elective caesarian section under more controlled circumstances and rapid volume replacement of the exsanguinated fetus. ${ }^{9}$

A prenatal diagnosis also implicates prolonged in-patient hospital admissions in the hope of optimizing fetal outcome. The correct time of admission should be well planned. Till date there is no agreed consensus about care for prenatally diagnosed cases. However once diagnosed it is considered reasonable to admit in hospital as early in third trimester, with a view to prompt caesarean section and access to neonatal care should membranes rupture. The cost effectiveness of approach is debatable. It is recommended that women should be offered elective delivery at 35 weeks or earlier after administration of corticosteroids for fetal lung maturity. ${ }^{9}$ In one series, $28 \%$ of elective admissions had emergency CS indicated for bleeding, rupture of membranes or labour. ${ }^{11}$ These 
authors believe that the mild risk associated with prematurity at 35 weeks is outweighed by risk of miserable outcome if membranes rupture, as in $8 \%$ of women membranes rupture before labour. Patients admitted electively must be counselled about the adverse outcome of unplanned emergency preterm delivery with significant morbidity and mortality, which in a small number of cases may occur even when they are being admitted and monitored. ${ }^{12}$ Antenatal corticosteroids should be considered at admission, the timing of which remains undetermined.

Planned care includes close surveillance and timed delivery. Once the diagnosis of vasa previa is made, the patient should be under continuous surveillance and admitted electively at 34 weeks. ${ }^{9}$ Cases should not be managed expectantly with labour occurring as these vessels may undergo compression by the presenting part during labour or may tear when membranes rupture leading to fetal exsanguination and death.

An innovative treatment has been reported by Ruben et al who could avoid these complications by obliterating these vessels in-utero by laser ablation. ${ }^{3}$ However this treatment has potential limitations. Accurate diagnosis is essential; as laser ablation of type 1 vasa previa may result in immediate fetal demise. Their successful management of the case highlights a potential role in optimizing management of type 2 vasa previa while decreasing hospital stay and resource expenditures.

It has been seen that routine screening can identify 1 to 2 cases of vasa previa in an average maternity unit in the UK every year. ${ }^{11}$ Sonographic prenatal diagnosis has the potential to dramatically improve perinatal outcome. Many authors recommend that standard obstetrical protocol should include screening for vasa previa and an attempt should be made to identify umbilical cord insertion..$^{8,12,13}$ The examination is not time consuming and is often accomplished in less than 1 minute. ${ }^{14}$ However, evidence to support the introduction of routine screening for vasa previa is currently limited. ${ }^{9}$ The outcome in cases which are diagnosed early in pregnancy is excellent. There is a higher chance of resolution when the condition is diagnosed before 24 weeks of gestation. ${ }^{15}$

\section{CONCLUSION}

Vasa previa is a rare but potentially fatal and unpredictable obstetrical disaster. Prenatal sonographic diagnosis has the potential to improve or prevent the poor obstetric and neonatal outcome associated with it.

Funding: No funding sources Conflict of interest: None declared

Ethical approval: Not required

\section{REFERENCES}

1. Datta S, Babu KM, Mitra S, Patil D. Vasa Previa: An Avoidable Obstetric Tragedy. J Obstet Gynaecol Ind. 2016;66(3):185-7.

2. Ruiter L, Kok N, Limpens J, Derks JB, de Graaf IM, Mol BW, et al. A systematic review on the diagnostic accuracy of ultrasound in the diagnosis of vasa previa. Ultrasound Obstet Gynecol. 2015;45: 516-22.

3. Fung TY, Lau TK. Poor perinatal outcome associated with vasa previa: is it preventable? A report of three cases and review of the literature. Ultrasou Obstet Gynecol. 1998;12(6):430-3.

4. Modanlou HD, Murata Y. Sinusoidal heart rate pattern: Reappraisal of its definition and clinical significance. J Obstet Gynaecol. 2014;30(3):169-80.

5. Sinkey RG, Odibo OA, Dashe J. Diagnosis and management of vasa previa. Am J Obstet Gynecol. 2015;37:615-9.

6. Sahoo G, Mohapatra I, Giri D. Study of maternal and perinatal outcome in antepartum hemorrhage. Ind J Prerinatalogy Reproduc Bio. 2012;2(2):27-9.

7. Othman M, Khojah S, Fathi T, Alkholy T, Aljayar L, Alhazmi J. Vasa praevia; case report. Webm Cent Obstet Gynaecol. 2014;5(5):WMC004643.

8. Lee W, Lee VL, Kirk JS, Sloan CT, Smith RS, Comstock $\mathrm{CH}$. Vasa previa: prenatal diagnosis, natural evolution, and clinical outcome. Obstet Gynecol. 2000;95(4):572-6.

9. Murray A, Murphy DJ. Vasa praevia: diagnosis and management. Obstet Gynaecol 2008;10:217-23.

10. Oyelese KO, Turner M, Lees C, Campbell S. Vasa previa: an avoidable obstetric tragedy. Obstet Gynecol Surv. 1999;54(2):138-45.

11. Oyelese Y, Catanzarite V, Prefumo F, Lashley S, Schachter M, Tovbin Y, et al. Vasa previa: the impact of prenatal diagnosis on outcomes. Obstet Gynecol. 2004;103(5):937-42.

12. Quintero RA, Kontopoulos EV, Bornick PW, Allen MH. In utero laser treatment of type II vasa previa. J Mater Fetal Neon Med. 2007;20(12),847-51.

13. Robert JA, Sepulveda W. Fetal exsanguination from ruptured vasa previa: still a catastrophic event in modern obstetrics. J Obstet Gynaecol. 2003;23(5):574.

14. Jantarasaengaram S, Suthipintawong C, Kanchana wat $S$, Thanagumtorn K. Ruptured vasa previa in velamentous cord insertion placenta. J Perinatol. 2007;27:457-9.

15. Erfani H, Haeri S, Shainker SA, Saad AF, Ruano R, Dunn TN, et al. Vasa previa: a multicenter retros pective cohort study. Am J Obstet Gynecol. 2019;221(6):644.e1-644.e5.

Cite this article as: Mohapatra I, Samantaray SR, Sindhuja VN. Vasa previa: A case report. Int J Reprod Contracept Obstet Gynecol. 2020;9:4301-3. 